\title{
AVALIAÇÃO DO IMPACTO DA CAPTAÇÃO D' ÁGUA NA LAGOA DO BONFIM, RN - BRASIL
}

\author{
Roberto Pereira ${ }^{1}$ \\ João Abner Guimarães Junior² \\ Gerson Cardoso da Silva Junior ${ }^{3}$ \\ Arthur Mattos ${ }^{4}$
}

\begin{abstract}
Resumo
O presente estudo compreende o desenvolvimento de um modelo computacional de balanço hídrico para simular o comportamento de um lago submetido a diferentes vazões de captação d'água, possibilitando a avaliação dos impactos efetivos associados ao processo de esvaziamento do reservatório. A metodologia desenvolvida foi aplicada à questão da avaliação dos impactos provocados pelo sistema adutor Trairi, na lagoa do Bonfim, maior reservatório do estado do Rio Grande do Norte. O modelo computacional foi desenvolvido em duas etapas: 1) determinação dos saldos de fluxo, o qual engloba os fluxos subterrâneos de entrada e saída da lagoa e outros consumos antrópicos (exceto o bombeamento da adutora) de difícil determinação direta, simplificando a abordagem do problema; 2) cálculo dos rebaixamento na lagoa associados com um determinado regime de vazões bombeadas. Os resultados mostraram que o volume captado para a adutora foi responsável por $48 \%$ do rebaixamento do nível d'água na lagoa do Bonfim no período de bombeamento, que foi de 1,17 m. O impacto seria muito maior se a vazão utilizada fosse, neste período, a prevista para o final do plano, o que implicaria num rebaixamento de $1,913 \mathrm{~m}$, correspondendo a aumento de $71,3 \%$ em relação ao rebaixamento observado.
\end{abstract}

Palavras-chave: Lago, Impacto ambiental

\footnotetext{
${ }^{1}$ CEFET-RN, Fone (0xx84) 215.2636, Fax (0xx184) 221.4005, E-mail: bobdudu@digi.com.br, Natal RN, Brasil.

2 UFRN/LARHISA/CT, Fone (0xx84) 215.3775, Fax (0xx84) 215.3703, E-mail: abner@ct.ufrn.br , Natal-RN, Brasil.

${ }^{3}$ I. GEOCIÊNCIAS-UFRJ, I. do Fundão - Rio de Janeiro, CEP: 21.949-900. Fone/Fax: (0xx21) 590.8091, E-mail: Gerson@acd.ufri.br, Rio de Janeiro-RJ, Brasil

${ }^{4}$ UFRN/LARHISA/CT, Fone (0xx84) 215.3775, Fax (0xx84) 215.3703, E-mail: armattos@ct.ufrn.br , Natal-RN, Brasil.
} 


\section{INTRODUÇÃO}

O presente estudo compreende o desenvolvimento de um modelo computacional de balanço hídrico para simular o comportamento de um lago submetido a diferentes vazões de captação d'água, possibilitando a avaliação dos impactos efetivos associado ao processo de esvaziamento do reservatório, o qual pode ser expresso pela redução do nível da superfície do espelho d'água.

A metodologia desenvolvida foi aplicada à questão da avaliação dos impactos provocados pelo sistema adutor Trairi, denominado de adutora Monsenhor Expedito, na lagoa do Bonfim, ponto de captação do sistema, o qual entrou em funcionamento em 01/08/98. O intervalo de estudo selecionado compreendeu os meses de novembro de 1998 até julho de 1999, justamente quando se implantou, neste trabalho, um monitoramento mais efetivo nesta lagoa, com a instalação de uma estação climatológica. Além disso, neste período a lagoa do Bonfim sofreu um rebaixamento expressivo e acelerado, indicando um quadro de possível comprometimento da disponibilidade hídrica local e das condições ambientais da área. Procurou-se, então, avaliar a parcela decorrente da captação da adutora e aquela gerada pelo déficit 76 hídrico que a região atravessou no período.

A lagoa do Bonfim, com uma área do espelho d'água de $9 \mathrm{~km}^{2}$ e uma capacidade de acumulação de 83 milhões de $\mathrm{m}^{3}$, é o maior reservatório da região litorânea oriental do estado do Rio Grande do Norte. Esta região possui as seguintes

características

hidrometeorológicas: clima tropical úmido (As' na escala Köpper), pluviometria média igual a $1.273 \mathrm{~mm}$ (SUDENE, 1990), com máximos valores ocorrendo principalmente nos meses de março a julho, e hidrografia pobre formada por rios perenes de pequeno porte.

A lagoa do Bonfim integra um sistema lacustre formado por seis lagoas (Figura 01), sendo as outras cinco (Redonda, Urubu, Boa Água, Ferreira Grande e Carcará) menores, conectado pelo aqüífero livre que é composto por rochas terciárias areno-argilosas da Formação Barreiras, associadas com uma cobertura arenosa eólica, do Quaternário (Pereira et alii, 1996). Informações potenciométricas (Pereira et alii, op. cit. e outros trabalhos subseqüentes) mostram que o setor oeste representa a principal zona de recarga da lagoa do Bonfim, sendo que esta repassa subterraneamente, e de forma divergente, em direção ao rio 
Pium (a norte), riacho Boacica (sudeste) em funcionamento, com uma vazão de e rio Trairi (a sul). cerca de $240 \mathrm{~L} / \mathrm{s}$. No final do plano, no

A adutora Monsenhor Expedito, horizonte de projeto no ano 2016, a com 315 Km de extensão, abastece 20 vazão deverá ser elevada para $430 \mathrm{~L} / \mathrm{s}$ municípios das regiões Trairi e Potengi beneficiando uma população de 222.336 do estado do Rio Grande do Norte. A habitantes

primeira etapa do projeto encontra-se

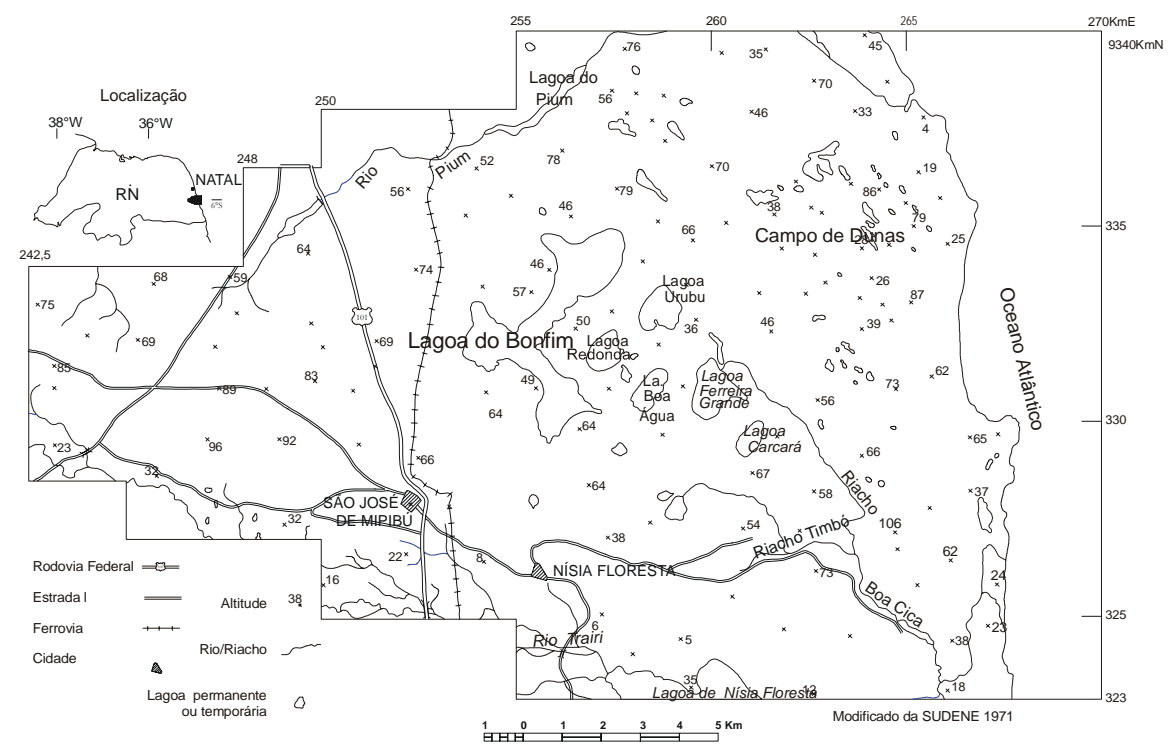

Figura 01 - Localização do sistema lacustre Bonfim.

\section{BALANÇO HÍDRICO DE LAGOS}

O balanço hídrico é um modelo conceitual de avaliação quantitativa dos recursos hídricos de uma região, relacionando os processos intervenientes no ciclo hidrológico.

Analiticamente, o balanço hídrico é expresso pela equação (1) diferencial geral da continuidade (Villela \& Mattos, 1975), baseada no princípio da conservação da massa de um sistema aplicado a um volume de controle, relacionando a taxa de variação do armazenamento interno com o saldo de fluxo externo no volume de controle.

$$
I-O=\frac{d S}{d t}
$$

Onde: I (input) e $O$ (output) são os totais de fluxos que entram e saem no volume de controle e $d s / d t$ é a taxa de variação do armazenamento interno no volume de controle.

$\mathrm{Na}$ equação (2) geral do balanço hídrico de um lago fechado (UNESCO, 1981), como é o caso da lagoa do Bonfim, os fluxos de entrada (I) compreendem (Figura 02): 0 
escoamento superficial (Qescoa), a espelho d' água $(A)$; as perdas recarga subterrânea do aqüífero subterrâneas do Lago ( $\left.Q_{\text {perdas}}\right)$ e os ( $Q_{\text {recarga }}$ ) e as precipitações pluviais $(P)$ consumo d'água ( $\left.Q_{\text {consumos }}\right)$. A taxa de sobre a área do espelho d'água $(A)$. Os variação do armazenamento $(d s / d t)$, em fluxos de saída $(O)$ são constituídos pela cada instante, pode ser avaliada pelo perda d'água por evaporação no produto da área do espelho d'água $(A)$ espelho, determinada pelo produto da pela taxa de variação do nível d'água do taxa de evaporação $(E)$ pela área do lago $(d h / d t)$.

$$
\left(P . A+Q_{\text {sup }}+Q_{\text {recarg } a}\right)_{\text {entrada }}-\left(E . A+Q_{\text {perdas }}+Q_{\text {consumos }}\right)_{\text {saidas }}=A \frac{d h}{d t}
$$

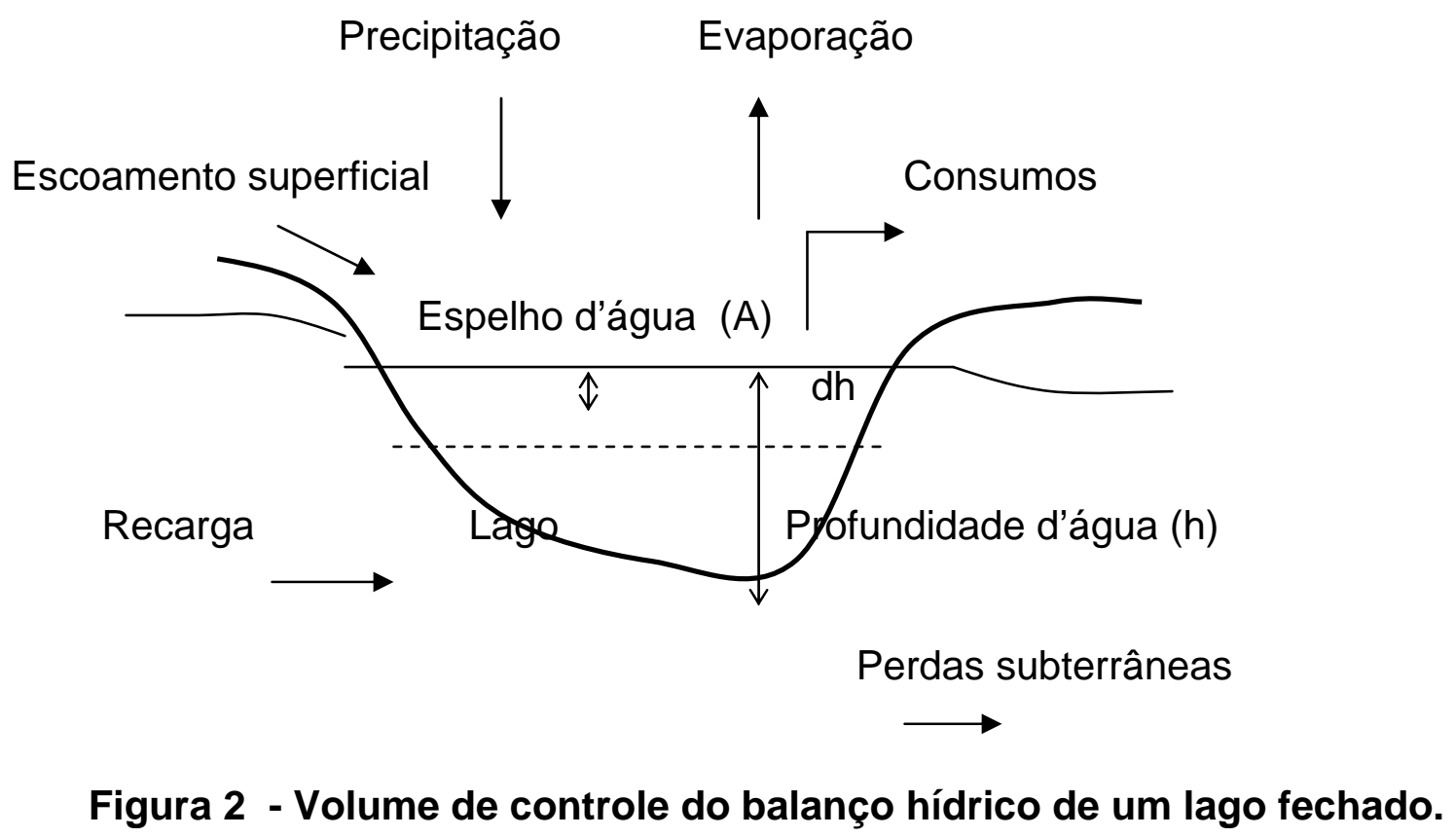

A área do espelho (A) pode ser expressa como função da profundidade (h), conforme a equação (3).

$$
A=k \cdot h^{\alpha}
$$

Onde: $A$ é a área do espelho d'água do reservatório, em $\mathrm{m}^{2} ; h(\mathrm{em} \mathrm{m})$ é a lâmina d'água, mas na simulação foi substituído pela altitude do nível do lagoa, como efeito prático, e $k$ e $\alpha$ são os coeficientes da função de regressão cota-área.

Para as condições da lagoa do Bonfim, o escoamento superficial direto pode ser desprezado, devido a não existência de uma rede de drenagem superficial direcionada para esta lagoa e o consumo d'água é expresso pela vazão bombeada pela adutora ( $\left.Q_{\text {bomba }}\right)$ e 
outros usos relevantes (Qoutros), tais Bonfim pode ser apresentada da como a irrigação. Portanto, a equação seguinte forma:

(4) do balanço hídrico da lagoa do

$$
\left(P . A+Q_{\text {rec arga }}\right)_{\text {entrada }}-\left(E . A+Q_{\text {perdas }}+Q_{\text {bomba }}+Q_{\text {outras }}\right)_{\text {saída }}=A \cdot \frac{d h}{d t}
$$

MODELO DE SIMULAÇÃO

Foi desenvolvido um modelo computacional de balanço hídrico mensal para simular o comportamento da lagoa do Bonfim durante o período observado de exploração, novembro/98 até julho/99, conforme inicialmente justificado.

O modelo teve como base os saldos de fluxos do balanço hídrico (termo residual) considerados como independentes do bombeamento, os quais foram determinados, inicialmente, a partir dos dados observados.

Para isto, contou-se com informações diárias de variação do nível d'água da lagoa, e, principalmente, com os dados de uma estação hidrometeorológica instalada às margens da referida lagoa, apresentando registros diários de precipitações pluviais, evaporação, velocidade e direção do vento, umidade relativa, temperatura do ar e pressão atmosférica.

\section{Estimativa do saldo de fluxo}

Os componentes do saldo de fluxo do balanço hídrico, definidos no contexto do estudo, constituem as recargas e as perdas subterrâneas da lagoa e os outros consumos antrópicos, os quais são apresentados na expressão (5). Sacks et al. (1992) e Sacks et al. (1998) consideraram, na aplicação de balanço hídrico de lagos, o termo residual como a diferença entre as entradas e saídas de águas subterrâneas para avaliar as trocas de águas subterrâneas com os lagos, na Flórida. É importante reconhecer que termos desconhecidos do balanço hídrico obtidos a partir de uma ou mais variáveis medidas, acumulam os erros destas.

$$
Q_{\text {saldo }}=Q_{\text {rec arga }}-\left(Q_{\text {perda }}+Q_{\text {outras }}\right)
$$

Combinado-se as equações (4) e (5), tem-se:

$$
Q_{\text {saldo }}=A \cdot \frac{d h}{d t}-p \cdot A+\left(E . A+Q_{\text {bomba }}\right)
$$

Discretizando-se a equação diferencial (6) pelo método das diferenças finitas, segundo um esquema progressivo no tempo, para uma área da superfície do espelho d'água média $(\bar{A})$, no intervalo de tempo $(\Delta t)$ mensal, chega-se a seguinte expressão: 
$Q_{\text {saldo }}^{t}=\bar{A}\left(h^{t+1}-h^{t}-P^{t}+E^{t}\right)+Q_{\text {bomba }}^{t}$

Onde: $Q_{\text {saldo }}^{t}$ e $Q_{\text {bomba }}^{t}$ são as vazões no mês atual $(t)$, em m³/mês; $h^{t}$ e $h^{t+1}$ são as profundidades d'água no inicio do mês atual e subseqüente, respectivamente, em m; $P^{t}$ e $E^{t}$ são, respectivamente, a precipitação pluvial e a evaporação no decorrer do mês atual, em m/mês. $\bar{A}$ é a área média mensal do espelho d'água, em $\mathrm{m}^{2}$, calculada a partir das áreas do espelho no início do mês atual e do mês subseqüente.

$$
\bar{A}=\frac{A^{t+1}+A^{t}}{2}
$$

\section{Simulação da lagoa do Bonfim}

O modelo de simulação determina as profundidades d'água na lagoa, no início de cada mês do período observado, a partir dos saldos de fluxo avaliados numa primeira etapa.

Substituindo-se na equação 6 a área A dada pela função (3) e desenvolvendo-se, chega-se a seguinte equação diferencial ordinária com relação à profundidade $h$.

$$
\frac{d h}{d t}=P-E+\frac{Q_{\text {saldo }}-Q_{\text {bomba }}}{k h^{\alpha}}
$$

Onde a derivada é uma função direta da profundidade d'água.

$$
\frac{d h}{d t}=f(h)
$$

Sendo:

$$
f(h)=P-E+\frac{Q_{\text {saldo }}-Q_{\text {bomba }}}{k h^{\alpha}}
$$

Onde: $\mathrm{h}$ é a profundidade d'água, em m; $P$ e E são, respectivamente, a precipitação pluviométrica e a evaporação, em m/mês; $Q_{\text {saldo }}$ e $Q_{\text {bomba }}$ são, respectivamente, o saldo de fluxo e a vazão bombeada, em $\mathrm{m}^{3} /$ mês e $k$ e $\alpha$ são os coeficientes da função de regressão cota-área.

Resolvendo-se, passo a passo, a equação diferencial com o auxílio do método de Runge-Kutta de $4^{\mathrm{a}}$ ordem (Ruggiero \& Lopes, 1988), para um intervalo de tempo " $\Delta t$ " mensal, chegase:

$$
h^{t+1}=h^{t}+\frac{1}{6}\left(k_{1}+2 k_{2}+2 k_{3}+k_{4}\right)
$$

Sendo :

$$
\begin{aligned}
& k_{1}=f\left(h^{t}\right) \\
& k_{2}=f\left(h^{t}+\frac{k_{1}}{2}\right) \\
& k_{3}=f\left(h^{t}+\frac{k_{2}}{2}\right) \\
& k_{4}=f\left(h^{t}+k_{3}\right)
\end{aligned}
$$


Onde: $h^{t}$ e $h^{t+1}$ são, respectivamente, as profundidades d'água, no início do mês atual e subseqüente, em $\mathrm{m}$.

\section{RESULTADOS E DISCUSSÃO}

Os resultados da metodologia proposta aplicada a lagoa do Bonfim, envolvendo todos os componentes do balanço hídricos, estão apresentados em quadros a seguir. Os saldos de fluxo do balanço hídrico mensal da lagoa, para as condições reais, foram determinados com o auxílio do Quadro 1 e o Quadro 2 mostra os rebaixamentos mensais da lagoa do Bonfim sem o efeito do bombeamento do sistema adutor.

Ainda, para avaliar a sustentabilidade hídrica da lagoa frente as demandas futuras da adutora, o modelo de simulação foi aplicado, no mesmo período estudado, para a vazão máxima de projeto no final do plano de 430 L/s ou 1.114 .560 m²/mês. O Quadro 3 mostra os resultados desta simulação.

Quadro 1 - Saldo de Fluxo do balanço hídrico da lagoa do Bonfim.

\begin{tabular}{|c|c|c|c|c|c|c|c|c|c|}
\hline Data & Prof. & Volume & Área & Precipitação & Evapo & ração & & Retiradas & \\
\hline $\mathrm{T}$ & $\begin{array}{l}\text { ht } \\
\text { (m) }\end{array}$ & $\begin{array}{c}V_{t} \\
\left(m^{3}\right)\end{array}$ & $\begin{array}{c}A_{t} \\
\left(m^{2}\right)\end{array}$ & $\begin{array}{c}\mathrm{P} \\
\mathrm{mm} / \mathrm{mês}\end{array}$ & $\begin{array}{c}\mathrm{E}_{\mathrm{t}} \\
\mathrm{mm} / \mathrm{mês}\end{array}$ & $\begin{array}{l}0,75 x E_{t} \\
\mathrm{~mm} / \mathrm{mês}\end{array}$ & $\begin{array}{c}\text { Vazão } \\
\text { bombeada } \\
\text { (m³/mês) }\end{array}$ & $\begin{array}{c}\text { Saldo de } \\
\text { Fluxo } \\
\text { (m²/mês) }\end{array}$ & $\begin{array}{c}\text { Total } \\
\text { (m³/mês) }\end{array}$ \\
\hline $01 / 11 / 98$ & 41,769 & 74.551 .857 & 8.202 .451 & 12,4 & 210,1 & 157,6 & 409.595 & 120.079 & 529.674 \\
\hline $01 / 12 / 98$ & 41,565 & 72.842 .258 & 8.052 .952 & 19,8 & 231,9 & 173,9 & 450.808 & 457.032 & 907.840 \\
\hline \begin{tabular}{|l|}
$01 / 01 / 99$ \\
\end{tabular} & 41,306 & 70.707 .738 & 7.865 .269 & 5,7 & 214,5 & 160,9 & 372.949 & \begin{tabular}{|l|}
297.247 \\
\end{tabular} & \begin{tabular}{|l|}
670.196 \\
\end{tabular} \\
\hline $01 / 02 / 99$ & 41,073 & 68.829 .952 & 7.699 .189 & 97,4 & 199,1 & 149,3 & 431.938 & 100.803 & 532.741 \\
\hline $01 / 03 / 99$ & 40,955 & 67.899 .576 & 7.616 .555 & 42,8 & 207,1 & 155,3 & 326.238 & 32.547 & \begin{tabular}{|l|}
358.785 \\
\end{tabular} \\
\hline $01 / 04 / 99$ & 40,800 & 66.689 .434 & 7.508 .722 & 138,6 & 201,2 & 150,9 & 693.617 & $\begin{array}{c}- \\
416.892\end{array}$ & 276.725 \\
\hline 01/05/99 & 40,753 & 66.320 .554 & 7.475.771 & 234,9 & 149,3 & 112,0 & 568.949 & $\begin{array}{c}- \\
241.528\end{array}$ & 327.421 \\
\hline 01/06/99 & 40,829 & 66.915 .352 & 7.528.883 & 102,3 & 160,6 & 120,5 & 539.737 & $\begin{array}{c}- \\
168.004\end{array}$ & 371.733 \\
\hline 01/07/99 & 40,764 & 66.407 .381 & 7.483.531 & 43,5 & 189,8 & 142,4 & 666.131 & $\begin{array}{c}- \\
137.320\end{array}$ & 528.811 \\
\hline $01 / 08 / 99$ & 40,599 & 65.144 .417 & 7.370 .457 & & & & & & \\
\hline Diferença & 1,170 & 9.407 .440 & Soma & 697,4 & $1.763,6$ & $1.322,7$ & 4.459 .962 & 43.964 & 4.503 .926 \\
\hline
\end{tabular}


Quadro 2 - Simulação operacional da lagoa do Bonfim sem bombeamento.

\begin{tabular}{|c|c|c|c|c|c|c|c|c|c|}
\hline Data & Prof. & Volume & Área & Precipitação & Evap & ração & & Retiradas & \\
\hline $\mathrm{t}$ & $\begin{array}{l}\text { ht } \\
(\mathrm{m})\end{array}$ & $\begin{array}{c}V_{t} \\
\left(m^{3}\right) \\
\end{array}$ & $\begin{array}{c}A_{t} \\
\left(m^{2}\right)\end{array}$ & $\begin{array}{c}\mathrm{P} \\
\mathrm{mm} / \mathrm{mês}\end{array}$ & $\begin{array}{c}\mathrm{E}_{\mathrm{t}} \\
\mathrm{mm} / \mathrm{mês}\end{array}$ & $\begin{array}{c}0,75 x E_{t} \\
\mathrm{~mm} / \mathrm{mês}\end{array}$ & \begin{tabular}{|c} 
Vazão \\
bombeada \\
(m³/mês)
\end{tabular} & $\begin{array}{c}\text { Saldo de } \\
\text { Fluxo } \\
\text { (m³/mês) }\end{array}$ & $\begin{array}{c}\text { Total } \\
\left(\mathrm{m}^{3} / \mathrm{mês}\right)\end{array}$ \\
\hline $01 / 11 / 98$ & 41,769 & 74.551 .857 & 8.202 .451 & 12,4 & 210,1 & 157,6 & & 120.079 & 120.079 \\
\hline $01 / 12 / 98$ & 41,614 & 73.249 .263 & 8.088 .608 & 19,8 & 231,9 & 173,9 & & 457.032 & 457.032 \\
\hline \begin{tabular}{|c|}
$01 / 01 / 99$ \\
\end{tabular} & 41,410 & 71.557 .037 & 7.940 .084 & 5,7 & 214,5 & 160,9 & & 297.247 & \begin{tabular}{|l|}
297.247 \\
\end{tabular} \\
\hline $01 / 02 / 99$ & 41,223 & 70.038 .095 & 7.806 .149 & 97,4 & 199,1 & 149,3 & & 100.803 & 100.803 \\
\hline $01 / 03 / 99$ & 41,161 & 69.533 .117 & 7.761 .489 & 42,8 & 207,1 & 155,3 & & 32.547 & 32.547 \\
\hline $01 / 04 / 99$ & 41,048 & 68.631 .325 & 7.681.567 & 138,6 & 201,2 & 150,9 & & $\begin{array}{c}- \\
416.892 \\
\end{array}$ & $\begin{array}{c}- \\
416.892 \\
\end{array}$ \\
\hline 01/05/99 & 41,088 & 68.953 .558 & 7.710.150 & 234,9 & 149,3 & 112,0 & & 241.528 & 241.528 \\
\hline 01/06/99 & 41,237 & 70.149.353 & 7.815.980 & 102,3 & 160,6 & 120,5 & & $\begin{array}{c}- \\
168.004\end{array}$ & \begin{tabular}{|c|}
- \\
168.004 \\
\end{tabular} \\
\hline 01/07/99 & 41,240 & 70.175 .476 & 7.818 .287 & 43,5 & 189,8 & 142,4 & & 137.320 & \begin{tabular}{c|}
- \\
137.320
\end{tabular} \\
\hline 01/08/99 & 41,162 & 69.542 .727 & 7.762 .339 & & & & & & \\
\hline Diferença & 0,607 & 5.009 .130 & Soma & 697,4 & $1.763,6$ & $1.322,7$ & - & 43.964 & 43.964 \\
\hline
\end{tabular}

Quadro 3 - Simulação operacional da lagoa do Bonfim para a vazão de final do plano.

\begin{tabular}{|c|c|c|c|c|c|c|c|c|c|}
\hline \multirow{2}{*}{$\begin{array}{c}\text { Data } \\
\mathrm{t}\end{array}$} & \multirow{2}{*}{$\begin{array}{l}\text { Prof. } \\
\text { ht } \\
(\mathrm{m})\end{array}$} & \multirow{2}{*}{$\begin{array}{c}\text { Volume } \\
V_{t} \\
\left(\mathrm{~m}^{3}\right)\end{array}$} & \multirow{2}{*}{$\begin{array}{c}\text { Área } \\
A_{t} \\
\left(m^{2}\right) \\
\end{array}$} & \multirow{2}{*}{$\begin{array}{c}\text { Precipitação } \\
P \\
\mathrm{~mm} / \mathrm{mês}\end{array}$} & \multicolumn{2}{|c|}{ Evaporação } & \multicolumn{3}{|c|}{ Retiradas } \\
\hline & & & & & $\begin{array}{c}E_{t} \\
\mathrm{~mm} / \mathrm{mês}\end{array}$ & $\begin{array}{l}0,75 x E_{t} \\
\mathrm{~mm} / \mathrm{mês}\end{array}$ & $\begin{array}{l}\text { CAERN } \\
\text { (m³/mês) }\end{array}$ & $\begin{array}{c}\text { Saldo de } \\
\text { Fluxo } \\
\text { (m³/mês) }\end{array}$ & $\begin{array}{c}\text { Total } \\
\text { (m³/mês) }\end{array}$ \\
\hline $01 / 11 / 98$ & 41,769 & 74.551 .857 & 8.202 .451 & 12,4 & 210,1 & 157,6 & 1.114 .560 & 120.079 & 1.234 .639 \\
\hline $01 / 12 / 98$ & 41,481 & 72.141 .756 & 7.991 .486 & 19,8 & 231,9 & 173,9 & 1.114 .560 & 457.032 & 1.57 \\
\hline 01/01/99 & 41,139 & 69.357 .416 & 7.745 .934 & 5,7 & 214,5 & 160,9 & 1.114 .560 & 297.247 & 1.41 \\
\hline $01 / 02 / 99$ & 40,809 & 66.761 .556 & 7.515 .160 & 97,4 & 199,1 & 149,3 & 560 & 100.803 & 1.2 \\
\hline 01/03/99 & 40,601 & 65.159 .689 & 7.371 .827 & 42,8 & 207,1 & 155,3 & 1.114 .560 & 32.547 & 1.147 .107 \\
\hline 01/04/99 & 40,340 & 63.192 .669 & 7.194 .817 & 138,6 & 201,2 & 150,9 & 1.114 .560 & $(416.892)$ & 697.668 \\
\hline $01 / 05 / 99$ & 40,234 & 62.406 .941 & 7.123 .793 & 234,9 & 149,3 & 112,0 & 1.114 .560 & $(241.528)$ & 873.032 \\
\hline 01/06/99 & 40,234 & 62.409 .616 & 7.124 .035 & 102,3 & 160,6 & 120,5 & 1.114 .560 & $(168.004)$ & 946.556 \\
\hline 01/07/99 & 40,087 & 61.334 .644 & 7.026 .565 & 43,5 & 189,8 & 142,4 & 1.114 .560 & $(137.320)$ & 977.240 \\
\hline 01/08/99 & 39,856 & 59.670 .325 & 6.874 .956 & & & & & & \\
\hline Diferença & 1,913 & 14.881 .531 & Soma & 697,4 & $1.763,6$ & $1.322,7$ & 10.031 .040 & 43.964 & 10.075 .004 \\
\hline
\end{tabular}

Os coeficientes das funções de Coeficientes da função de regressão regressão cota-área e cota-volume, cota-área: $A=k_{1} \cdot h^{\alpha}$ apresentados em seguida, formam determinada a partir dos dados do levantamento batimétrico realizado pela COSTA (1997).

$\mathrm{k}_{1}=6,466720$

$\alpha=3,765459$

Coeficiente de correlação $=0,988580$ 
Coeficientes da função de regressão cota-volume: $\mathrm{V}=\mathrm{k}_{2} \cdot \mathrm{h}^{\beta}$

$\mathrm{K}_{2}=1,496567$

$\beta=4,748954$

Coeficiente de correlação $=$ 0,998692

O Quadro 4, seguinte, apresenta uma síntese comparativa dos resultados encontrados nas diversas condições de bombeamento estudadas para a lagoa do Bonfim.

A fragilidade da lagoa do Bonfim, frente à explotação do período analisado, pode também ser constatada considerando que o volume positivo resultante do somatório do saldo de fluxo, em torno de $+43.964 \mathrm{~m}^{3}$ (Quadro 1), o qual corresponde apenas ao fluxo subterrâneo de entrada que fica disponível no lago, é bastante pequeno em relação ao volume bombeado pela adutora que foi de $4.459 .962 \mathrm{~m}^{3}$, lembrando que para a vazão de final de plano, o volume a ser explotado, seria em torno de 10.031.040 $\mathrm{m}^{3}$ (Quadro 3).

\section{Quadro 4 - Síntese das condições de bombeamento na lagoa do Bonfim.}

\begin{tabular}{|c|c|c|c|c|c|c|}
\hline $\begin{array}{c}\text { Condição de } \\
\text { bombeamento }\end{array}$ & $\begin{array}{c}\text { Volume da Lagoa } \\
\text { no final do período }\end{array}$ & Desnível & Variação de volume & \multicolumn{2}{c|}{$\begin{array}{c}\text { Volume } \\
\text { bombeado }\end{array}$} \\
\cline { 2 - 7 } & $\mathrm{m}^{3}$ & $\%^{1}$ & $\mathrm{~m}$ & $\mathrm{~m}^{3}$ & $\mathrm{~m}^{3}$ & $\%^{2}$ \\
\hline Sem & 69.542 .727 & 93,3 & 0,607 & 5.009 .130 & 0 & 0 \\
\hline Real & 65.144 .417 & 87,4 & 1,170 & 9.407 .440 & 4.459 .962 & 47,4 \\
\hline Final do plano & 59.670 .325 & 80,0 & 1,913 & 14.481 .531 & 10.031 .040 & 69,3 \\
\hline
\end{tabular}
$2 \%$ relativa ao volume da lagoa no início do bombeamento $\left(74.551 .857 \mathrm{~m}^{3}\right)$
\% relativa a variação de volume da lagoa no período.

\section{CONCLUSÕES}

O modelo simplificou abordagem do problema englobando no saldo de fluxo os componentes do balanço hídrico de difícil determinação direta, tais como os fluxos subterrâneos de entrada e saída da lagoa do Bonfim e outros consumos. Entretanto, para a aplicação desta metodologia requer dados mínimos de monitoramento da pluviometria, evaporação e nível d`água.
O volume captado para a Adutora a foi responsável por $48 \%$ do rebaixamento do nível d'água na lagoa do Bonfim no período de bombeamento, que foi de 1,17 m, mostrando, portanto, que a captação é um fator muito impactante no sistema lacustre.

O impacto devido ao bombeamento seria muito maior se a vazão utilizada fosse, neste período, a prevista para o final do plano, o que 
implicaria num rebaixamento de 1,913

$\mathrm{m}$, correspondendo a aumento de $71,3 \%$

em relação ao rebaixamento observado.

\section{Agradecimentos}

Os autores agradecem ao Centro Federal de Educação Tecnológica do Rio Grande do Norte (CEFET), a Universidade Federal do Rio Grande do Norte (UFRN) e a Universidade Federal do Rio de Janeiro (UFRJ).

\section{REFERÊNCIAS BIBLIOGRÁFICAS}

COSTA - Consultoria e Serviços Técnicos e Ambientais Ltda/SERHID - 1997. Estudos da Disponibilidade Hídrica da Lagoa do Bonfim.

PEREIRA, R.; CHIAVENATO, M. C.; FREITAS, J.; REIS, L. M. M. 1996. Carta Geotécnica do Município de Nísia Floresta - Rn. In: SIMPÓSIO BRASILEIRO DE CARTOGRAFIA GEOTÉCNICA, 2, São Carlos, 1996. Boletim...,ABGE, p.233-239.

RUGGIERO, M. A. G.; LOPES, V. L. R. 1988. Cálculo Numérico. Aspectos Teóricos e Computacionais. São Paulo, McGraw-Hill.

SACKS, L. A.; LEE, T. M.; TIHANSKY, A. B. 1992. Hydrogeologic and preliminary data analysis for the hydrologic-Budget assessment of lake Barco, an acidic seepage lake in the Putnam County, Florida. Water-Resources Investigations, 91-4180.

SACKS, L. A.; SWANCAR, A.; LEE, T. M. 1998. Estimating ground-water exchange with lakes using water-budget and chemical mass-balance approaches for ten lakes in ridge areas of Polk and Highlands Counties, Florida. Water-Resources Investigations, 98-4133.

SUDENE 1990. Dados Pluviométricos Mensais do Nordeste. Recife (Série Pluviométrica , 4).

UNESCO 1981. Methods of Computation of the Water Balance of Large Lakes and Reservoirs. Fontenoy, Paris. 122 p.

VILLELA, S.M.; MATTOS, A. 1975. Hidrologia Aplicada. São Paulo, McGraw-Hill do Brasil. 245 p. 